\title{
SUPPORTING DELEGATION IN SECURE WORKFLOW MANAGEMENT SYSTEMS
}

\author{
Vijayalakshmi Atluri ${ }^{1}$, Elisa Bertino ${ }^{2}$, Elena Ferrari ${ }^{3}$ \\ and Pietro Mazzoleni ${ }^{2}$ \\ ${ }^{1}$ MSIS Department and CIMIC, Rutgers University, atluri@ cimic.rutgers.edu \\ 2 DiCo Department, University of Milan, bertino,mazzoleni@dsi.unimi.it \\ ${ }^{3}$ SSCCFFMM Department, University of Insubria, Italy, Elena.Ferrari@uninsubria.it
}

\begin{abstract}
Workflow systems are today used in numerous business application domains including office automation, finance and banking, as well as in scientific application domains, for automating their day-to-day applications, Often, organizations establish a set of security policies that regulate how the business process and resources should be managed. For reasons of ease in management, these security policies are expressed in terms of roles. In addition to simple authorization rules specifying which subject/role can execute a task in a workflow, many business processes require support for more complex authorization constraints, such as separation of duties. In this paper, we present an approach that supports delegation and assign users to roles in such a way that no constraints are violated. In particular, we introduce the notion of delegation consistency and propose algorithms to assign tasks to users such that they guarantee delegation consistency.
\end{abstract}

Keywords: Access control, Workflow Systems, Delegation

\section{Introduction}

Workflow management aims at modeling and controlling the execution of business processes involving a combination of manual and automated activities in an organization. Workflow systems are today used in numerous business application domains including office automation, finance and banking, as well as in scientific application domains such as in spatial processes and DNA sequencing, for automating their day-today applications. A workflow is defined as a set of coordinated activities that achieves a common business objective [1]. An activity, or a task, is a logic step or description of a piece of work that contributes toward the accomplishment of a process $[7,5]$. Tasks that build up the workflow are usually related and dependent upon one another, which in turn are spec- 
ified by task dependencies. A workflow management system (WFMS) is a system that supports process specification, enactment, monitoring, coordination and administration of workflow process through the execution of software, whose order of execution is based on the workflow logic [1].

The desired security to ensure the secrecy, correctness and integrity are adhered to a business process and are specified through a set of security policies, which are commonly used to define constraints that regulate how the business process and resources should be managed. For reasons of ease in management, often these security policies are expressed in terms of roles. In addition to simple authorization rules specifying which subject/role can execute a task in a workflow, many business processes require support for more complex authorization constraints, such as separation of duties (SOD) [4, 8].

In [2], Bertino et al. present a language to express these different types of authorization constraints as clauses in a logic program, and propose solutions to verify the consistency of the constraint specification and to assign users and roles to tasks of the workflow in a such a way that no constraints are violated. In this paper, we extend the work presented in [2] to support delegation in secure workflow systems. The basic idea behind delegation is that an active entity in a system, called delegator delegates authority to another active entity, called delegatee, to carry out some functions on behalf of the former. In the context of workflow systems, delegation amounts to transfer of duties of executing a task within a workflow. We call this task the delegated task.

In case of delegation, one must ensure that no authorization constraints are violated due to the delegation activity. We illustrate the problem of the inconsistencies due to the interplay between authorization constraints and delegation by considering a simple example.

Example 1 Let $W$ be a workflow, which represents the process of reviewing a paper for a conference, consisting of three tasks $\left\{T_{1}, T_{2}, T_{3}\right\}$

Assume $T_{1}$ refers to submitting a paper, $T_{2}$ refers to preparing three reviews, and $T_{3}$ refers to collecting the reviews and making the final decision to accept or reject the paper. The obvious dynamic separation of duties constraints in this example include: (1) Though anyone among the set of reviewers may be a reviewer, for any given paper, the three reviewers have to be different individuals, and (2) an author cannot be the reviewer for his own paper. Now suppose that a user, John, wishes to delegate the duty assigned to him to one of his colleagues (Jane). Such a delegation is legitimate and may prove valuable as Jane may have better expertise on the topic of the paper. However, if the delegation is entertained without taking the authorization constraints into account, a violation of the constraint may arise. A violation occurs if John is 
assigned to review a paper coauthored by Jane, and he further wishes to delegate it to Jane.

This paper is organized as follows. Section 2 reviews the workflow model and the authorization rules proposed in [2]. Section 3 presents our workflow delegation model. Section 4 provides an overview of the different phases of our approach, that are delegation consistency analysis phase, planning phase, and runtime phase which are further described into Section 5,6 , and 7 respectively. Section 8 concludes the paper and outlines future work.

\section{Authorization Constraints in Workflow}

\subsection{The Workflow model}

As in most WFMSs, we assume that a workflow consists of several tasks to be executed sequentially.

Each task is associated with one or more roles, which are the only ones authorized to execute the task. In the remainder of this paper, $U$, $\mathcal{R}$ and $\mathcal{T}$ denote the set of users, the set of roles, and the set of tasks in a given workflow, respectively. Additionally, $U_{i}$ denotes the set of users authorized to play role $R_{i}$. We refer to the association of roles with tasks in a workflow as workflow role specification, formally defined as follows.

Definition 1 (Workflow Role Specification) A workflow role specification $W$ is a list of task role specifications $\left[\mathrm{TRS}_{1}, \mathrm{TRS}_{2}, \ldots, \mathrm{TRS}_{n}\right.$ ], where each $\mathrm{TRS}_{i}$ is a triple $\left(T_{i}, R S_{i}\right.$, act $\left._{i}\right)$ where $T_{i} \in \mathcal{T}$ is a task, $R S_{i} \subseteq \mathcal{R}$ is the set of roles authorized to execute $T_{i}$ and act $t_{i} \in \Gamma$ is the number of possible activations of task $T_{i}$.

In the following, we use the term workflow and workflow role specification as synonyms. A workflow may have several workflow instances. We assume that each instance inherits the same workflow role specification from the workflow from which it is generated.

\subsection{Constraint language}

Authorization constraints, used to express security policy, are defined as clauses in a normal logic program [6]. Such language is not intended as the end-user language for expressing constraints but it is internally used by the system to make it easy for analyzing and enforcing constraints. However, a visual programming environment can be developed on top of this language along the lines of the system discussed in [3]. In [2], authors present a constraint language semantics to formalize specific information about the workflow. Such semantics is built upon sets of 
predicate symbols denoted as specification $(\mathrm{SP})$, execution (EP), planning (PP) and comparison (CP) predicates. Constraints are expressed as rules of a logic program. An authorization rule $a r$ is an expression of the form: $H \leftarrow A_{1}, \ldots, A_{n}$, not $B_{1}, \ldots$, not $B_{m}, n, m \geq 0$, where $H, A_{1}, \ldots, A_{n}$ and $B_{1}, \ldots, B_{m}$ are atoms and not denotes negation by failure [6]. $H$ is the rule head, whereas $A_{1}, \ldots, A_{n}$, not $B_{1}, \ldots, B_{m}$ is the rule body.

For instance, the rule $a r_{1}=$ cannot_do do $_{u}\left(U_{i}, \mathrm{~T}_{2}\right) \leftarrow$ execute ${ }_{u}\left(U_{i}, \mathrm{~T}_{2}, k\right)$, states that if user $U_{i}$ has been assigned more than $k$ times for executing task $T_{2}$, it cannot be assigned any longer for $T_{2}$.

We call Authorization Constraint-Base of a workflow W $(A C B(W))$, the set of rules specifying all the authorization constraints associated with $W$. Given $A C B(W)$ for a workflow $W$, it is possible to compute the set of roles/users that must be prevented/obliged to execute a task $T_{i}$. In the rest of the paper, we denote as Denied_Roles $\left(T_{i}\right)$, Denied_Users $\left(T_{i}\right)$, Obliged_Roles $\left(T_{i}\right)$, Obliged_Users $\left(T_{i}\right)$ these sets.

\section{The Workflow Delegation Model}

\subsection{Delegation Predicates}

In this section, we formally introduce the delegation predicates, $\mathcal{D} \mathcal{P}$, as the set of predicates used to specify the different types of task delegations in a workflow. We categorize them into the following two types - delegation predicates (BP) and volunteer delegation predicates (VP) where $\mathcal{D P}=\mathcal{B P} \cup \mathcal{V P}$.

Delegation Predicates: The predicates belonging to $\mathcal{B P}$ contain delegation predicates as well as the predicates for rejecting delegation. They comprise delegate.tu $\left(U_{i}, T_{k}, U_{j}\right)$, by which a user $U_{i}$ delegates to user $U_{j}$ all the instances of task $T_{k}$ assigned to him/her by the WFMS, delegate_tu $\left(U_{i}, T_{k}\right)$ by which $U_{i}$ expresses the intention to delegate $T_{k}$ without specifying the delegatee user, reject_del_t $\left(U_{i}, T_{k}\right)$ by which $U_{i}$ rejects instances of $T_{k}$ delegated to him/her by any other user and finally reject_del_tu $\left(U_{i}, T_{k}, U_{j}\right)$ rejecting only $T_{k}$ delegated to $U_{i}$ from $U_{j}$.

Moreover, $\mathcal{B P}$ contains two additional predicates: delegate_ru $\left(R_{i}, T_{k}, U_{j}\right)$ and delegate_ $r r\left(R_{i}, T_{k}, R_{j}\right)$ that, differently from the others, cannot be specified by any workflow user, but are reserved to the workflow administrator. These predicates specify that the instances of task $T_{k}$ assigned to users belonging to role $R_{i}$ are redirected to user $U_{j}$ or any users belonging to role $R_{j}$. Those predicates allow the administrator to temporarily change the possible task instance assigned to a role without modifying the basic authorizations. 
Volunteer Predicates: Similar to the delegation predicates, it is possible to define volunteer predicates, $\mathcal{V P}$.

Volunteer predicate, volunteer_t $\left(U_{i}, T_{k}, n\right)$ allows a user $\left(U_{j}\right)$ to nominate himself for the execution of a certain number $(n)$ of instances of a task $\left(T_{k}\right)$ that have been delegated by another user, perhaps based on certain incentives he/she may receive due to such an activity. We provide this capability because we believe that it is not realistic for a user to nominate himself only for tasks delegated by a specific user. To make this specification more realistic, we enhance the semantics of the language with a parameter that allows the user to set a maximum limit on the number of delegated tasks he wishes to volunteer for.

\subsection{Delegation Rules}

We use the formalism introduced in section 2 and the delegation predicates $(\mathcal{D P})$ to define delegation rules. Formally:

Definition 2 (Delegation Rule) A delegation rule is of the form $\mathrm{dr}$ : $H \leftarrow L_{1}, \ldots, L_{n}$, where $H$ is a delegation predicate and $L_{i}, i=1 \ldots n$ is either an execution or a delegation literal.

Given a delegation rule $d r$, in the following we will use Delegator $(d r)$, Delegatee $(d r)$ and Task $(d r)$ to refer to the Delegator User, the Delegatee User and the Delegated Task of the delegation predicates in the left hand side of $d r$, respectively.

Definition 3 (Constraint-Base) Let $W$ be a workflow. The Delegation Constraint-Base associated with $W$ (written $D C B(W)$ ) is the set of delegation rules. The constraint base of $W$, denoted as $\mathcal{C B}(\mathcal{W})=$ $A C B(W) \cup D C B(W)$.

\section{Overview of our Approach}

In this section, we provide a roadmap of the methodology adopted in this paper to support the issue of delegation in secure workflow systems so that no authorization constraints are violated. Specifically, we address the problem of assigning roles and users to tasks of a workflow such that both authorization and delegation rules are satisfied. Our methodology is organized according to three phases, as shown in Figure 1.

1 Static Analysis Phase: In this phase, we analyze the consistency of the delegations. The consistency analysis is in turn organized done in two levels, internal and external. The internal analysis is to determine if there are inconsistencies in the delegation rule base (i.e., $D C B(W)$ ) itself. External analysis is to determine if the del- 


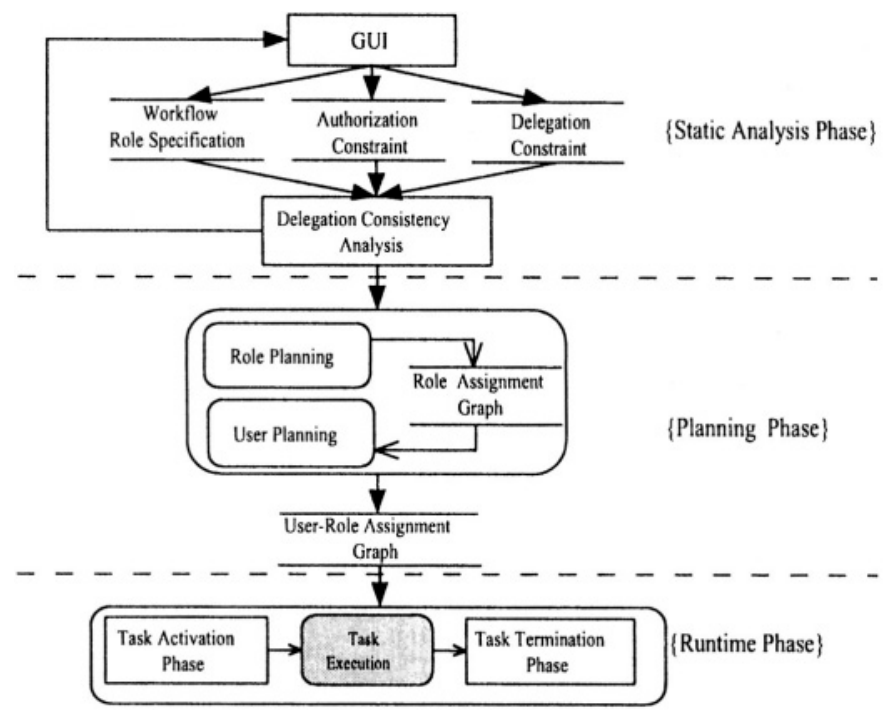

Figure 1. Phases in constraints authorization analysis and enforcement

egation rules can be enforced without causing any inconsistencies when authorization rules are also enforced.

2 Planning Phase: The goal of this phase is to generate role and user assignments to tasks before the workflow execution. Note that while much of the role-task assignment can be done during this phase, a complete user-task assignment cannot be done prior start executing the workflow. This phase comprises of two sub-phases: (i) The Role Planning Phase, in which we employ an exhaustive algorithm to generate a Role Assignment Graph $(R A G(W))$ representing all possible role assignments for each task in the workflow. (ii) The User Planning Phase, in which we employ a heuristic approach that plans assignments of users based on $R A G(W)$ and authorization rules in $A C B(W)$. We denote the graph generated as a result of this process by User Role Assignment Graph, $(U R A G(W))$.

3 Runtime Phase: The goal of this phase is to select an authorized user according to the rules in $\mathcal{C B}(\mathcal{W})$. In the sub-phase, referred to as Task Activation Phase, the delegation rules are enforced and the inconsistencies among rules are resolved. Then, the task can be executed by the assigned user. The runtime phase, which is executed for each task of the workflow, is terminated with the

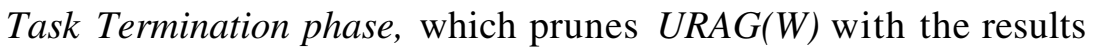


of the task execution and prepares the system for subsequent task assignments.

\section{Delegation Consistency Checking}

In Section 3, we have defined delegation as a set of logic constraints. Here, we develop the formalism to help verify the consistency of these rules with respect to the overall workflow specification. This analysis is carried out statically as well as during the runtime. The Static Analysis, addressed in this section, aims at resolving conflicts that arise independently from a specific instance of the workflow. This check can be done statically in the sense that it does not depend on the execution history of the workflow. The analysis conducted during the runtime phase identifies and resolves inconsistencies arising for a particular assignment of users to tasks in a workflow. We address this runtime evaluation part in Section 7.

Intuitively, $\mathcal{C B}(\mathcal{W})$ is said to be statically consistent with respect to delegation, if all the delegation rules do not result in violation of any authorization constraints. The first type of static inconsistency arises when there is a cycle in the specification of delegations. A delegation cycle occurs when users' intentions to delegate a task creates an infinite loop. Although it is not an inconsistency in its strictest sense, it is important to identify such condition as well because it effects the feasibility of executing a workflow.

In addition to identifying cycles in delegation rules, the static analysis check aims at evaluating inconsistencies that arise among delegation and authorization constraints in the workflow. For example, assume the rules cannot_do $o_{u}\left(J_{o h n}, T_{1}\right) \leftarrow$ and delegate_tu $\left(J a n e, T_{1}\right.$, John $) \leftarrow$ belong to the model of a $\mathcal{C B}(\mathcal{W})$. In this case, an inconsistency can be statically discovered since John cannot be delegated for $T_{1}$ under any circumstances given that he is not allowed to execute $T_{1}$. In order to verify the consistency among the delegation rules and the authorization rules, i.e., in $\mathcal{C B}(\mathcal{W})$, the following concepts are introduced. These define the set of possible users that can delegate, volunteer or being delegated for a given task $T_{i}$ :

- Delegator_Users $\left(T_{i}\right)=\bigcup\left\{u_{j} \mid\right.$ delegate $t\left(u_{j}, T_{i}\right) \in \mathcal{C B}(\mathcal{W})$ OR delegate_tu $\left.\left(u_{j}, T_{i}, u_{i}\right) \in \mathcal{C} \mathcal{B}(W)\right\}$ Delegator_Users $\left(T_{i}\right)$ represents the set of users who wish to delegate the execution of $T_{i}$ according to the rules in $\mathcal{C B}(\mathcal{W})$;

- Delegatee_Users $\left(T_{i}\right)=\bigcup\left\{u_{j} \mid\right.$ delegate_tu $\left.\left(u_{i}, T_{i}, u_{j}\right) \in \mathcal{C B}(\mathcal{W})\right\}$ Delegatee_Users $\left(T_{i}\right)$ represents the set of users who have $T_{i}$ delegated by some other users according to the rules in $\mathcal{C B}(\mathcal{W})$; 
- Volunteer_Users $\left(T_{i}\right)=\bigcup\left\{u_{j} \mid\right.$ volunteer_tu $\left.\left(u_{j}, T_{i}, u_{i}\right) \in \mathcal{C B}(\mathcal{W})\right\}$ Volunteer_Users $\left(T_{i}\right)$ represents the set of users who volunteer themselves for the execution of instances of $T_{i}$, according to the rules in $\mathcal{C B}(\mathcal{W})$;

It is important to ensure that users who cannot execute a task are not allowed to delegate, being delegated and volunteer for the execution of the task. Furthermore, it is necessary to verify that users who are supposed to execute a task should not delegate it to some other user. The above requirements are formalized in the following definition.

\section{Definition 4 (Delegation Consistency)}

We say that $\mathcal{C B}(\mathcal{W})$ is consistent with respect to delegation if and only if the following is verified: $\forall T_{i} \in W$,

- If Obliged_Users $\left(T_{i}\right) \neq \emptyset$, then (Delegator_Users $\left(T_{i}\right) \cap$ Delegatee_Users $\left.\left(T_{i}\right)\right)$ - Obliged_Users $\left(T_{i}\right)$ $=\emptyset$;

- If Denied_Users $\left(T_{i}\right)=\emptyset$, then Denied_Users $\left(T_{i}\right) \cap$ Delegatee_Users $\left(T_{i}\right)=\emptyset_{;}$and

- Denied_Users $\left(T_{i}\right) \cap$ Volunteer_Users $\left(T_{i}\right)=\emptyset$.

\section{Planning phase}

The planning phase generates the set of possible roles and user assignments to the workflow tasks so that all the authorization constraints are satisfied. This phase is performed statically, i.e. before the workflow execution starts, and aims at both providing an initial plan and reducing, as much as possible, the number of operations to be accomplished by the system at runtime. Planning is accomplished in two subphases: role planning phase and user planning phase.

\subsection{Role Planning phase}

Our approach to identify role assignments for a given workflow $W$ is similar to the solution proposed in [2]. It begins with the assumption that the number of roles associated with a task is not large. Therefore, an exhaustive approach is taken where all the possible combinations of roles assigned to the tasks are evaluated against the authorization constraints. To keep role planning feasible, we assume all the task activations be executed by the same role. However, at runtime we relax this assumption by letting different task activations assigned to different users. The result of this phase is the Role Assignment Graph, $R A G(W)$, defined as follows: 


\section{Definition 5 (Role Assignment Graph)}

Let $W=\left[\mathrm{TRS}_{1}, \mathrm{TRS}_{2}, \ldots, \mathrm{TRS}_{n}\right]$ be a workflow role specification with TRS $_{i}=\left(T_{i},\left(R S_{i}, \succ_{i}\right), a c t_{i}\right), i=1, \ldots, n$. The Role Assignment Graph of $W(\mathrm{RAG}(\mathrm{W}))$ is a labeled graph $G=(V, E)$ defined as follows:

- Vertices. There is a vertex labeled $\left(T_{i}, R_{j}\right), T_{i} \in \mathrm{TRS}_{i}, R_{j} \in R S_{i}$ only if the assignment of role $R_{j}$ to task $T_{i}$ does not violate the authorization constraints. A vertex labeled $\left(T_{i}, R_{j}\right)$ thus states that role $R_{j}$ can be assigned to task $T_{i}$ during the workflow execution.

- Edges. There exists an arc connecting $\left(T_{i}, R_{j}\right)$ to $\left(T_{h}, R_{k}\right)$ only if assigning task $T_{i}$ to a user belonging to role $R_{j}$ and assigning task $T_{h}$ to user belonging to $R_{k}$ for the same workflow instance do not violate any constraints.

$R A G(W)$ contains a path $\left\{\left(T_{1}, R_{1}\right) \ldots\left(T_{n}, R_{n}\right)\right\}$ if and only if the assignments of $R_{i}$ to $T_{i}, i=1 \ldots n$ do not violate any workflow constraint.

Example 2 Consider a workflow $\mathrm{W}$ with three tasks $T=\left(T_{1}, T_{2}, T_{3}\right)$ that can be executed by users belonging to roles $\left\{R_{a}, R_{b}, R_{c}\right\}$, respectively. Suppose $\mathcal{C B}(\mathcal{W})$ contains the following rules:

$a r_{1}=$ cannot_dor $\left(R_{c}, T_{1}\right) \leftarrow$,

$a r_{2}=$ cannot $_{-} d o_{r}\left(R_{a}, T_{3}\right) \leftarrow$ execute $_{r}\left(R_{a}, T_{1}, 1\right)$,

$a r_{3}=$ cannot_do $o_{r}\left(R_{c}, T_{2}\right) \leftarrow$, and

$a r_{4}=$ cannot_do $_{r}\left(R_{b}, T_{3}\right) \leftarrow$ execute $_{r}\left(R_{b}, T_{2}, 1\right)$ RAG $(\mathrm{W})$ generated according to Definition 5 is as shown in Figure 2. The selected nodes and paths are all the ones consistent with respect to the rules in $\mathcal{C B}(\mathcal{W})$. For instance, there is no edge between $\left(T_{2}, R_{b}\right)$ and $\left(T_{3}, R_{b}\right)$ in $\mathrm{RAG}(\mathrm{W})$ since it conflicts with rule $a r_{4}$.

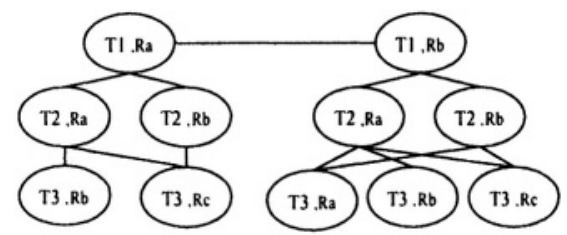

Figure 2. the $R A G(W)$ generated for the workflow in to Example 2

\subsection{User Planning Phase}

In a workflow, it is quite common that the number of users is much larger than the number of roles. Specifically, the number of users assigned to a role significantly increases as we traverse from higher level roles to lower level roles in the role hierarchy. The exhaustive strategy 
described in Section 6.1, can no longer be applied to user planning since the number of possible users assigned to a task can be very high. Therefore, we chose to execute user planning completely at runtime. However, even though we do not do user planning in advance, we statically enhance $R A G(W)$ with more information to speed up the runtime phase. To accomplish this, we introduce User-Role Assignment Graph, URAG(W). Essentially, $U R A G(W)$ is an enhanced and sometimes instantiated version of $R A G(W)$ with information concerning which users belonging to a given role must or must not be assigned for the execution of a task. Briefly, in this phase, we evaluate rules which are not influenced by the workflow execution so they can be verified in advance, and if it is the case that a user belonging to a role $R_{j}$ must execute a task $T_{k}$, then each node $\left(T_{k}, R_{j}\right)$ in $R A G(W)$ is updated with such information. We denote the node representing such users with ' + '. Analogously, each node in $R A G(W)$ is complemented with information about the set of users that must be prevented from executing that task, according to the constraints specified by the static rules. We denote the node representing such users with '-'. Formally, $\operatorname{URAG(W)}$ can be defined as follows:

Definition 6 (User-Role Assignment Graph) Let RAG(W) be a Role Assignment Graph for a workflow $W=\left[\mathrm{TRS}_{1}, \mathrm{TRS}_{2}, \ldots, \mathrm{TRS}_{n}\right]$, where each $\mathrm{TRS}_{i}=\left(T_{i},\left(R S_{i}, \succ_{i}\right)\right.$, act $\left._{i}\right), i=1, \ldots, n$. Let $C B_{S}(W)$ be the set of static rules belonging to $\mathcal{C B}(\mathcal{W})$. The User-Role Assignment Graph of $W$, URAG(W), is a labeled graph $G=(V, E)$ defined as follows:

\section{- Vertices.}

There exists a vertex labeled $v_{k}=\left(T_{i}, R_{j}\right.$, users, value $)$ for each $\left(T_{i}, R_{j}\right) \in R A G(W)$ where users and value are defined as follows:

- If Auth User $=\left\{u \mid\right.$ must_execute $_{u}\left(u, T_{i}\right) \leftarrow \operatorname{belong}\left(u, R_{j}\right) \in$ $\left.C B_{S}(W)\right\} \neq \emptyset$ then users $:=$ Auth $_{U \text { ser }}$, value $=+$; Otherwise

- If Auth $_{\text {User }}=\left\{u \mid\right.$ cannot_do $\left(u, T_{i}\right) \leftarrow$ belong $\left(u, R_{j}\right)$

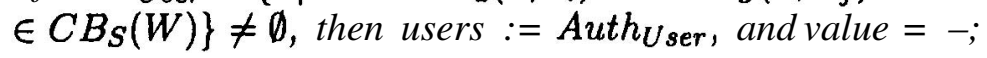

- If cases 1 and 2 do not hold, then users $=\emptyset$, and value $=-$.

\section{- Edges.}

There exists an arc from $v_{m}=\left(T_{i}, R_{j}\right.$, users $\left(v_{m}\right)$, value $\left.\left(v_{m}\right)\right)$ to $v_{n}=\left(T_{h}, R_{k}\right.$, users $\left(v_{n}\right)$, value $\left.\left(v_{n}\right)\right)$ only if there exists an arc from $\left(T_{i}, R_{j}\right)$ to $\left(T_{h}, R_{k}\right)$ in RAG $(\mathrm{W})$.

Example 3 Consider the workflow of Example 2, and assume that $\mathcal{C B}(\mathcal{W})$ contains the static rules 
$r_{1}=\left(\left(\right.\right.$ must_execute ${ }_{u}\left(u_{i}, T_{2}\right) \leftarrow$ belong $\left(u_{i}, R_{A}\right), u_{i}=\{$ John, Jane $\left.\}\right)$, and $r_{2}=\left(\left(\right.\right.$ cannot_do $o_{u}\left(u_{i}, T_{3}\right) \leftarrow$ belong $\left(u_{j}, R_{C}\right), u_{j}=\{$ Bob, Bill $\left.\}\right)$ in the model. The resulting $\mathrm{URAG}(\mathrm{W})$, generated according to Definition 6 , is presented in Figure 3.

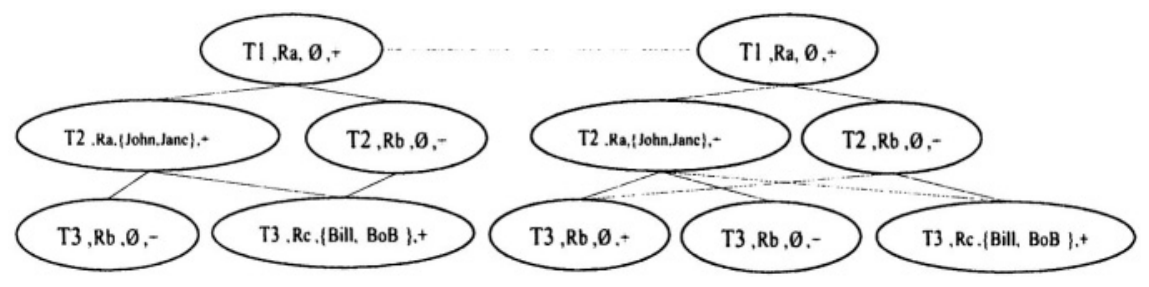

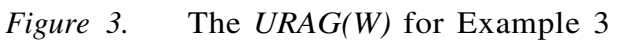

\section{Runtime Phase}

The runtime phase is executed upon task activation and termination. It consists of two phases: the Task activation phase executed before the actual execution of the task to define users assigned for carrying out the activities, and the Task termination phase, executed after the termination of the task to update the workflow with the task's execution results and to prepare $U R A G(W)$ for subsequent task assignments.

Task activation phase: The check in this phase entails verifying several conditions in order to identify the user authorized to execute a task $T_{j}$. In this phase a user (or several users in case of multiple task execution) is assigned to task $T_{j}$. First, this phase identifies a candidate role among the ones authorized to execute $T_{j}$. We call $n_{\text {ass }}$ the node and Role $\left(n_{a s s}\right)$ its referred node. Second, from Role $\left(n_{a s s}\right)$ the system selects a possible candidate user $\left(u_{a s s}\right)$. Two cases are possible here: if value $\left(n_{a s s}\right)=+$, then $u_{\text {ass }}$ has to be selected from users $\left(n_{\text {ass }}\right)$; if value $\left(n_{\text {ass }}\right)=-$; otherwise $u_{\text {ass }}$ needs to be picked from all the users belonging to Role $\left(n_{\text {ass }}\right)$ except from the ones in users $\left(n_{\text {ass }}\right)$. Finally, before sending $T_{i}$ to $u_{\text {ass }}$ for execution, the system needs to verify that no inconsistencies arise between $u_{a s s}$ and rules in $\mathcal{C B}(\mathcal{W})$ having the same task in the head and in one of the execution literals of the body (such as example cannot_do $\left(B o b, T_{i}\right) \leftarrow$ execute $\left.e_{u}\left(B o b, T_{i}\right)\right)$. Those constraints, defined as Recursive rules in the following, cannot be evaluated before or after the process of assigning tasks to user in a workflow.

To evaluate a rule in Recursive $\left(T_{i}\right)$, we use an hypothetical reasoner. For example, to determine whether the execution of the task $T_{i}$ by user $u_{j}$ would violate Recursive $\left(T_{i}\right)$, we insert into $\mathcal{C B}(\mathcal{W})$ the rules, called Assignment Hypotheses, execute $u_{u}\left(u_{j}, T_{i}, k\right) \leftarrow$ and $\operatorname{success}\left(T_{i}, k\right) \leftarrow$. 
There rules allow evaluation of Recursive $\left(T_{i}\right)$. If successful, the candidate user is entitled to execute $T_{i}$ and the hypotheses can be removed from $\mathcal{C B}(\mathcal{W})$. Otherwise, $u_{j}$ is not authorized to execute $T_{i}$ and the process continues selecting another user among the ones available.

Task Termination phase: This is performed after task execution. During this phase, information about the user who has executed the task is inserted into $\mathcal{C B}(\mathcal{W})$. Rules

execute $_{r}\left(\operatorname{Role}\left(n_{a s s}\right), T_{j}, k\right) \leftarrow$ and execute ${ }_{u}\left(u_{a s s}, T_{j}, k\right) \leftarrow$ are added to $\mathcal{C B}(\mathcal{W})$ and used by the system to update nodes in $U R A G(W)$ upon the evaluation of $\operatorname{Simple}\left(T_{j}\right)$ using the same methodologies presented in section 6.2.

Delegation Rule Application. The peculiarity of delegation rules, which are a special case of recursive rules as described before, is that these rules can only be evaluated after a user has been actually assigned for the execution of a task. In other words, until the assignment process is finished where a user $u_{i}$ assigned for $T_{j}, u_{i}$ 's (Delegator) intention to delegate $T_{j}$ to some other user $u_{k}$ (Delegatee) cannot take place. The reason why the system should be prevented from applying delegation in advance, is to eliminate the possibility of overcoming the authorization system by delegating a task to an unauthorized user.

Different assumptions can be made in case of role-delegation since they are defined only by the workflow administrator. To improve the performance of the assignment process, their evaluation is applied as soon as a Delegator Role is selected by the system as a candidate for executing the task.

The process of evaluating user delegation rules, is applied as soon as a user has been successfully assigned for executing a task. It starts with a check as to whether a delegating rule exists in the model which formalizes the user intentions to delegate the task. If such rule exists, the system first identifies a delegatee user and then verifies that the delegation does not violate any recursive rules. To determine a delegatee user, we provide three progressive solutions. First we look at the user specified in the delegating rule. If delegatee is not in the rule or the user is not authorized for the task, we examine the volunteer users. Finally, if the model does not contain any volunteer user, we extend the search to all the users authorized for the task. 


\section{Conclusions and Future Research}

In this paper, we have presented an approach that provides support for delegation in workflow systems in such a way that no authorization constraints are violated while entertaining delegation.

Currently, we are working on several extensions to this work, which are briefly explained below. We are currently extending our work to include several types of conditions that specify under which circumstances a delegation can occur (such as time related and workload related situations). In fact, while this paper proposes a solution where delegation is considered for all the instances of a given task, we are aware that in reality a person might wish to nominate someone for a work under certain circumstances. However, even if we did not present this here, our approach is carved in such a way that it can be easily extended to address these different types of conditions. Additionally, we plan on extending the our approach to verify if a given task can be delegated or not and when the right to delegate can be transmitted to another user.

We intend to introduce the concept of Delegation Access Control associated with a task that is independent from the workflow. This will enable delegation to be enforced over several workflows, including interorganizational processes, in a decentralized manner.

\section{References}

[1] Workflow reference model. Technical report, Workflow Management Coalition, Brussels, 1994.

[2] Elisa Bertino, Elena Ferrari, and Vijayalakshmi Atluri. An Approach for the Specification and Enforcement of Authorization Constraints in Workflow Management Systems. ACM Transactions on Information Systems Security, 2(1), February 1999.

[3] S.K. Chang, G. Polese, R. Thomas, and S. Das. A Visual Language for Authorization Modeling. In Proc. of the IEEE Symposium on Visual Languages (VL97), 1997.

[4] David D. Clark and David R. Wilson. A comparison of commercial and military computer security policies. In Proc. IEEE Symposium on Security and Privacy, pages 184-194, Oakland, California, April 1987.

[5] Dimitrios Georgakopoulos, Mark Hornick, and Amit Sheth. An Overview of Workflow Management: From Process Modeling to Workflow Automation Infrastructure. Distributed and Parallel Databases, pages 119-153, 1995.

[6] J.W. Lloyd. Foundations of Logic Programming. Springer-Verlag, 1984.

[7] Marek Rusinkiewicz and Amit Sheth. Specification and Execution of Transactional Workflows. In W. Kim, editor, Modern Database Systems: The Object Model, Interoperability, and Beyond. Addison-Wesley, 1994.

[8] R. Sandhu. Separation of Duties in Computerized Information Systems. In Database Security IV: Status and Prospects, pages 179-189, 1991. 